\title{
Nanoparticle sensor for label free detection of swine DNA in mixed biological samples.
}

\begin{abstract}
We used $40 \pm 5 \mathrm{~nm}$ gold nanoparticles (GNPs) as colorimetric sensor to visually detect swine-specific conserved sequence and nucleotide mismatch in PCR-amplified and nonamplified mitochondrial DNA mixtures to authenticate species. Colloidal GNPs changed color from pinkish-red to gray-purple in $2 \mathrm{mM}$ PBS. Visually observed results were clearly reflected by the dramatic reduction of surface plasmon resonance peak at $530 \mathrm{~nm}$ and the appearance of new features in the $620-800 \mathrm{~nm}$ regions in their absorption spectra. The particles were stabilized against salt-induced aggregation upon the adsorption of singlestranded DNA. The PCR products, without any additional processing, were hybridized with a 17-base probe prior to exposure to GNPs. At a critical annealing temperature $\left(55^{\circ} \mathrm{C}\right)$ that differentiated matched and mismatched base pairing, the probe was hybridized to pig PCR product and dehybridized from the deer product. The dehybridized probe stuck to GNPs to prevent them from salt-induced aggregation and retained their characteristic red color. Hybridization of a 27-nucleotide probe to swine mitochondrial DNA identified them in porkvenison, pork-shad and venison-shad binary admixtures, eliminating the need of PCR amplification. Thus the assay was applied to authenticate species both in PCR-amplified and non-amplified heterogeneous biological samples. The results were determined visually and validated by absorption spectroscopy. The entire assay (hybridization plus visual detection) was performed in less than $10 \mathrm{~min}$. The LOD (for genomic DNA) of the assay was $6 \mu \mathrm{g} \mathrm{ml}-$ 1 swine DNA in mixed meat samples. We believe the assay can be applied for species assignment in food analysis, mismatch detection in genetic screening and homology studies between closely related species.
\end{abstract}

Keyword: Nanoparticle sensor; Detection. 